\title{
Dust and Hco+gas in the Star-forming Core W3-se
}

\section{Citation}

Zhu, Lei, M. C. H. Wright, Jun-Hui Zhao, and Yuefang Wu. 2010. "Dust and Hco+gas

in the Star-forming Core W3-se." The Astrophysical Journal 712 (1): 674-84. https://

doi.org/10.1088/0004-637x/712/1/674.

\section{Permanent link}

http://nrs.harvard.edu/urn-3:HUL.InstRepos:41555827

\section{Terms of Use}

This article was downloaded from Harvard University's DASH repository, and is made available under the terms and conditions applicable to Other Posted Material, as set forth at http:// nrs.harvard.edu/urn-3:HUL.InstRepos:dash.current.terms-of-use\#LAA

\section{Share Your Story}

The Harvard community has made this article openly available.

Please share how this access benefits you. Submit a story.

\section{Accessibility}




\title{
DUST AND HCO+ GAS IN THE STAR-FORMING CORE W3-SE
}

\author{
Lei ZhU ${ }^{1,3}$, M. C. H. Wright ${ }^{2}$, Jun-Hui ZhaO ${ }^{1}$, ANd Yuefang Wu ${ }^{3}$ \\ ${ }^{1}$ Harvard-Smithsonian Center for Astrophysics, 60 Garden Street, Cambridge, MA 02138, USA; lzhu@ @fa.harvard.edu \\ ${ }^{2}$ Department of Astronomy, University of California, Berkeley, Berkeley, CA 94720, USA \\ ${ }^{3}$ Department of Astronomy, Peking University, Beijing, China \\ Received 2009 June 24; accepted 2009 December 22; published 2010 March 5
}

\begin{abstract}
We report new results from recent Combined Array for Research in Millimeter-wave Astronomy (CARMA) observations of both continuum and $\mathrm{HCO}^{+}(1-0)$ line emission at $\lambda 3.4 \mathrm{~mm}$ from W3-SE, a molecular core of intermediate mass, together with the observations of continuum emission at $\lambda 1.1$ and $\lambda 0.85 / \lambda 0.45 \mathrm{~mm}$ with the Submillimeter Array (SMA) and the James Clerk Maxwell Telescope, respectively. A continuum emission core elongated from SE to NW, with a size of $\sim 10^{\prime \prime}$, has been observed at the millimeter and submillimeter wavelengths. The dust core has been resolved into a double source with the SMA at $\lambda 1.1 \mathrm{~mm}$. The angular separation between the two components is $\sim 4^{\prime \prime}$. Together with the measurements from the Spitzer Space Telescope and the Midcourse Space Experiment at mid-IR wavelengths, we determined the spectral energy distribution (SED) of the continuum emission from W3-SE and fit it with a thermal dust emission model. Our best fitting of the SED suggests the presence of two dust components with different temperatures. The emission at millimeter/submillimeter wavelengths is dominated by a major component that is characterized by a temperature of $T_{\mathrm{d}}=41 \pm 6 \mathrm{~K}$ with a mass of $65 \pm 10 \mathrm{M}_{\odot}$. In addition, there is a weaker hot component $(\sim 400 \mathrm{~K})$ which accounts for emission in the mid-IR, suggesting that a small fraction of dust has been heated by newly formed stars. We also imaged the molecular core in the $\mathrm{HCO}^{+}(1-0)$ line using CARMA at an angular resolution $\sim 6^{\prime \prime}$. In the central region of $\sim 50^{\prime \prime}$, the integrated $\mathrm{HCO}^{+}(1-0)$ line emission shows a main component $\mathrm{A}$ that coincides with the dust core, as well as two substructures $\mathrm{B}$ and $\mathrm{C}$ which are located $\mathrm{N}$ and SE of the dust core, respectively. With the CARMA observations, we have verified the presence of a blue-dominated double peak profile toward this core. The line profile cannot be explained by infall alone. The broad velocity wings of the line profile suggest that other kinematics such as outflows within the central $6^{\prime \prime}$ of the core likely dominate the resulting spectrum. The kinematics of the substructures of B and C suggest that the molecular gas outside the main component A appears to be dominated by the bipolar outflow originated from the dust core with a dynamical age of $>3 \times 10^{4}$ yr. Our analysis, based on the observations at wavelengths from millimeter, submillimeter, to mid-IR, suggest that the molecular core W3-SE hosts a group of newly formed young stars and protostars.
\end{abstract}

Key words: ISM: clouds - ISM: jets and outflows - ISM: kinematics and dynamics - ISM: molecules - radio lines: ISM - stars: formation

\section{INTRODUCTION}

Among the dynamical processes in star formation regions, infall appears to be critical in understanding the processes of mass accumulation in star formation cores and the growth of protostars. The protostellar infall rate depends on the surface density of the protostellar core, which usually can be determined from observations of self-absorptions of the embedded molecular clumps in it. However, it is difficult to unambiguously identify infall based on low angular-resolution observations because the spectral features of a complex with bipolar molecular outflows, rotating structures, or multiple cores can produce similar characteristics to those expected from self-absorption in infalling clumps. In some young star formation regions that show spectral signatures of infall from single-dish observations, the single-dish spectral features often turn out to be contaminated by outflows and other kinematic features with further high-resolution observations using interferometer arrays.

W3-SE is a dense molecular core located $2 \mathrm{kpc}$ away (Blitz et al. 1982), SE of the well-known massive star formation region W3-Main. The W3-SE core was first imaged by Tieftrunk et al. (1998) in the $\mathrm{NH}_{3}(1,1)$ and $(2,2)$ lines. The low kinetic temperature of $25 \mathrm{~K}$ based on the $\mathrm{NH}_{3}$ line ratio (Tieftrunk et al. 1998) and the absence of IRAS emission (Jijina et al. 1999) implied that this core is probably in an early evolutionary stage. A virial mass of $\sim 300 M_{\odot}$ was inferred from the line widths, indicating that this core may be a birthplace for intermediateor high-mass stars. However, to date there has been no evidence for $\mathrm{OB}$ stars present in the core because of the non-detection from the radio continuum emission at $6 \mathrm{~cm}$ with the Very Large Array (VLA; Tieftrunk et al. 1998). On the other hand, a possible infall signature in W3-SE was suggested by $\mathrm{Wu}$ et al. (2007) based on observations of multiple molecular lines with the IRAM $30 \mathrm{~m}$ telescope. Both the $\mathrm{HCO}^{+}(1-0)$ and (3-2) lines show an asymmetric double profile characterized by a primary peak blueshifted with respect to a peak velocity of the optically thin lines such as $\mathrm{C}^{18} \mathrm{O}(1-0)$, while a secondary peak is redshifted. The possible signature of infall in W3-SE suggested from the single-dish observations needs further verification with improved, high-resolution observations. Besides, Ruch et al. (2007) reported a detection of a mid-IR point source in the region of W3-SE and determined the flux densities in the four bands of the Infrared Array Camera (IRAC) on the Spitzer Space Telescope. The color-color diagram in their paper indicates that this source is most likely to be a young stellar object (YSO; Class I or earlier) with large excess emissions at longer wavelengths.

In this paper, we present new results based on observations at $\lambda 3.4,1.1,0.85 / 0.45 \mathrm{~mm}$ with the Combined Array for 
Table 1

Calibrators for the CARMA and SMA Observations

\begin{tabular}{|c|c|c|c|c|c|c|c|}
\hline \multirow[t]{2}{*}{ Date } & \multirow{2}{*}{$\begin{array}{c}\lambda \\
(\mathrm{mm})\end{array}$} & \multicolumn{2}{|c|}{ Bandpass } & \multicolumn{2}{|c|}{ Phase } & \multicolumn{2}{|c|}{ Flux Density } \\
\hline & & Source & Flux (Jy) & Source & Flux (Jy) & Source & Flux (Jy) \\
\hline 2008 Jun 24 & 3.4 & $3 \mathrm{C} 84$ & 6.1 & $0102+584$ & 2.1 & Uranus & 7.2 \\
\hline 2008 Jun 27 & 3.4 & $3 \mathrm{C} 454.3$ & 27.8 & $0102+584$ & 2.5 & MWC349 & 1.3 \\
\hline 2008 Oct 27 & 1.1 & $3 \mathrm{C} 454.3$ & 13.2 & $0244+624$ & 0.6 & Uranus & 45.6 \\
\hline
\end{tabular}

Research in Millimeter-wave Astronomy (CARMA), the Submillimeter Array (SMA), ${ }^{4}$ and the James Clerk Maxwell Telescope (JCMT), ${ }^{5}$ respectively, along with the data at mid-IR wavelengths obtained from both the Spitzer Space Telescope and Midcourse Space Experiment (MSX) archives. Section 2 describes observations and data reductions, Section 3 presents results from the analysis of the continuum emission at multiple wavelengths and the CARMA observations of the $\mathrm{HCO}^{+}(1-0)$ line, and Section 4 discusses the results and their implications for astrophysics. Finally, Section 5 gives the summary and conclusions.

\section{OBSERVATIONS AND DATA REDUCTION}

\subsection{CARMA Observations at $\lambda 3.4 \mathrm{~mm}$}

W3-SE was observed at $\lambda 3.4 \mathrm{~mm}$ with the CARMA telescope in the D-configuration on 2008 June 24 and June 27, with an angular resolution of $\sim 6^{\prime \prime}$. The observations were centered at R.A.(J2000) $=02^{\mathrm{h}} 25^{\mathrm{m}} 54.50$, decl. $(\mathrm{J} 2000)=62^{\circ} 04^{\prime} 11^{\prime \prime}$.0, which is used as the reference position in the following analysis. Two broad bands with $\mathrm{BW}$ of $500 \mathrm{MHz}$ were set at 86 and 89 $\mathrm{GHz}$ to image the continuum emission. Two upper side band (USB) narrow bands with $\mathrm{BW}$ of 8 and $32 \mathrm{MHz}$ were tuned to the $\mathrm{HCO}^{+}(1-0)$ line at $89.188 \mathrm{GHz}$, and the two lower side band (LSB) narrow bands were used for the $\mathrm{SiO}(2-1)$ line at $86.243 \mathrm{GHz}$. The highest velocity resolution is $0.42 \mathrm{~km} \mathrm{~s}^{-1}$ for the $8 \mathrm{MHz}$ narrow-band data with 63 spectral channels. The observations were Doppler-tracked at $V_{\mathrm{LSR}}=-38.9 \mathrm{~km} \mathrm{~s}^{-1}$.

The observations include two tracks. The first one lasted for $5 \mathrm{hr}$ using 14 antennas with a 19 point mosaic, and the second one was taken for $4 \mathrm{hr}$ using 13 antennas with a 7 point mosaic. Because the first track was on a maintenance day, several antennas were not used in parts of the track. The corrupted UV data were flagged during the off-line data reduction. The second track was carried out in good weather, and the data quality is excellent with $T_{\text {sys }}$ between 150 and $200 \mathrm{~K}$.

All the data reductions and imaging processes were carried out in MIRIAD (Sault et al. 1995). The data from the two tracks were calibrated separately with the calibrators listed in Table 1. The bandpass and complex gain calibrations were processed following the standard procedure for the CARMA data reduction. Flux density scales were calibrated using Uranus (7.2 Jy) and MWC 349 (1.3 Jy) at $\lambda 3.4 \mathrm{~mm}$ for the two tracks, respectively. After calibration the two data sets were merged and images were made with a linear mosaic algorithm using the program INVERT.

\footnotetext{
4 The SMA is a joint project between the Smithsonian Astrophysical Observatory and the Academia Sinica Institute of Astronomy and Astrophysics and is funded by the Smithsonian Institution and the Academia Sinica.

5 The James Clerk Maxwell Telescope is operated by the Joint Astronomy Centre on behalf of the Particle Physics and Astronomy Research Council of the UK, the Netherlands Organization for Scientific Research, and the National Research Council of Canada.
}

\subsection{Combining CARMA Data with IRAM $30 \mathrm{~m}$ Data}

The shortest baseline of the CARMA observations is $2.7 \mathrm{k} \lambda$. To eliminate the effects of missing short spacing, we combined the CARMA and IRAM $30 \mathrm{~m}$ telescope data which cover the central $45^{\prime \prime} \times 75^{\prime \prime}$ region (Wu et al. 2007). We used the MIRIAD program IMMERGE to linearly combine the single-dish image and the cleaned CARMA image. The UV ranges sampled by the two observations are well overlapped, which are used to determine the scaling factor for the flux density of the singledish data. The amplitude of the IRAM $30 \mathrm{~m}$ data was re-scaled by applying the scaling factor determined by comparing the amplitude values of the overlapped visibilities in the fast Fourier transform (FFT) domain. There is a boundary effect on the edge of the combined image of $45^{\prime \prime} \times 45^{\prime \prime}$ due to limited spatial coverage of the IRAM observations. The effect is negligible in our analysis of the line emission in the central $20^{\prime \prime} \times 20^{\prime \prime}$ region.

\subsection{SMA Observations at $\lambda 1.1 \mathrm{~mm}$}

We also made observations at $\lambda 1.1 \mathrm{~mm}$ with the SMA in the compact array with an angular resolution of $\sim 2$ ". 5. The SMA observations were centered at the same position as that used in the CARMA observations. The frequencies were tuned to 266.7/276.7 GHz for LSB/USB with bandwidths of $2 \mathrm{GHz}$ for each of the side bands. The typical $T_{\text {sys }}$ in the observations is 180-200 K.

The data reduction and imaging were made using MIRIAD. The bandpass shapes and complex gains were calibrated using QSOs 3C454.3 and 0244+624, respectively. The flux density scale was bootstrapped from Uranus using the SMA planet model. The continuum image of W3-SE was constructed by combining the visibility data from the two side bands with a rms noise of $1.5 \mathrm{mJy}$.

\subsection{JCMT Observations at $\lambda 0.85 / \lambda 0.45 \mathrm{~mm}$}

The W3-SE region was observed using JCMT with SCUBA at $\lambda 0.85$ and $0.45 \mathrm{~mm}$ on 2004 September 15 (PID M04BC17). The data were calibrated and combined into intensity images with the ORAC-DR pipeline. The FWHM beam sizes of the JCMT images are 14.5 and 7..5 at $\lambda 0.85$ and $\lambda 0.45 \mathrm{~mm}$, respectively. The JCMT images were re-gridded with the program REGRID in MIRIAD to align the coordinate axes with those in the CARMA images.

\subsection{Spitzer and MSX Data at Mid-IR}

The entire region of W3 was observed by Gehrz (the PI in GTO program PID 127) with the IRAC (Fazio et al. 2004) on 2004 January 10 and the Multiband Imaging Photometer for Spitzer (MIPS; Rieke et al. 2004) on 2004 January 10 on board the Spitzer Space telescope. The results have been published by Ruch et al. (2007), and a point source that is detected in all four IRAC bands in the W3-SE region was reported. In order to search for additional weaker mid-IR sources 
to identify the possible counterparts of the radio sources that have been detected at millimeter/submillimeter wavelengths, we have further investigated the W3-SE region by re-reducing the archive data of IRAC and MIPS (GTO program PID 127).

The basic calibrated data (BCD) products of IRAC are provided by the Spitzer Science Center (SSC) with the IRAC pipeline version S18.7.0. A post-BCD script $h d r \_$mask.pl provided by SSC was used to fix the potential saturation problem. The background correction and mosaic were made with the version 18.2.0 of the post-BCD reduction software MOPEX (Makovoz \& Khan 2005), producing a mosaic image with 1".22 per pixel. The point-source extraction and the aperture photometry were done with the package APEX in MOPEX. Point-spread function (PSF) fittings were carried out in the process of detecting point-like sources, and those with signal-to-noise ratio higher than 6 are identified as point sources. An aperture with radius of $2 . \prime 4$ and an annulus between $2 . .4$ and $7 " .3$ were used for the aperture photometry to determine the flux densities of the point sources. Aperture correction, color correction, and pixelphase correction were carried out according to the procedures described in the IRAC Data Handbook (ver. 3.0). In addition, the flux density of the W3-SE core was determined using the procedures of aperture photometry for extended sources with a radius of 8.5 in order to compare with the flux densities of millimeter/ submillimeter in the same region and to determine the SED. We noted that it was difficult to make a reliable annulus to represent the background levels due to the background/foreground stars. Therefore, to avoid obvious stellar emission, we measured flux densities in several regions which are close to W3-SE to calculate the background levels. An overall fractional error of $\sim 15 \%$ was assessed for the photometry, dominated by the uncertainties in the corrections for the background/foreground emission levels $(\sim 10 \%)$ and the aperture correction $(\sim 10 \%)$.

The BCD products of MIPS were pre-processed by the MIPS pipeline version $\mathrm{S} 16.1 .0$. The $\mathrm{W} 3-\mathrm{SE}$ region is badly saturated in the 70 and $160 \mu \mathrm{m}$ images due to the strong emission of W3-Main; thus, only the $24 \mu \mathrm{m}$ data were used. A post-BCD script flatfield.pl provided by SSC was used to make an extra flatfielding to remove the potential residual background gradient, and then the background correction and mosaic were made with MOPEX. The aperture photometry was carried out with the program aperture in MOPEX with an aperture radius of 13.'5, and color/aperture corrections were made following the MIPS Data Handbook (ver. 3.3.1). An uncertainty of $15 \%$ in the flux densities is dominated by the errors in corrections for the background/foreground emission $(\sim 10 \%)$ and the color/ aperture corrections.

In addition, we also investigated the $M S X$ images of W3-SE in all the four bands $(8.3,16.7,12.1$, and $21.3 \mu \mathrm{m})$ with an angular resolution of $\sim 20^{\prime \prime}$. We re-grided all of the Spitzer and MSX images in MIRIAD to align the coordinate axes with those in the CARMA images. The photometry was carried out with the similar methods as those for the IRAC images. The uncertainties of the photometry on the MSX images are large $(\sim 40 \%)$ due to poor sensitivities, low angular resolutions, and severe contamination from background/foreground emission.

\section{RESULTS}

\subsection{Continuum Emission}

3.1.1. CARMA $\lambda 3.4 \mathrm{~mm}$

Figure 1(a) shows the CARMA image of W3-SE at $\lambda 3.4 \mathrm{~mm}$, revealing an un-resolved dust core plus a tail-like structure extended in SE. We determined a peak position $(\delta$ (R.A.) $=$ $-5.5 \pm 0^{\prime \prime} .2$ and $\delta($ decl. $\left.)=-0.9 \pm 0.2\right)$ and peak intensity $(24 \pm$ $2 \mathrm{mJy}$ beam $^{-1}$ ) for the continuum emission from the dust core based on a Gaussian fitting to the data. Integrated over the core region of $15^{\prime \prime} \times 15^{\prime \prime}$, a total flux density of $42 \pm 10 \mathrm{mJy}$ was determined.

\subsubsection{SMA $\lambda 1.1 \mathrm{~mm}$}

Figure 1(b) shows the SMA image at $\lambda 1.1 \mathrm{~mm}$. The dust core has been resolved into two continuum emission components (SMA-1 and 2), and the overall morphology of the W3-SE dust core shows an elongation from NW to SE. The primary (SMA-1) and secondary (SMA-2) components of the double source are located NW and SE of the CARMA peak position. From fits with two Gaussian components and a linear background, we obtained peak intensities of $0.21 \pm 0.01$ and $0.17 \pm 0.01 \mathrm{Jy} \mathrm{beam}^{-1}$ for SMA-1 and SMA-2, respectively. The angular separation of the double components is $\sim 4^{\prime \prime}$. The total flux densities and sizes of the double components are listed in Table 2. The peak position of the emission at $\lambda 3.4 \mathrm{~mm}$ observed with CARMA agrees well with the centroid position of the double components observed with the SMA. We integrated the continuum flux density from the core in the SMA $1.1 \mathrm{~mm}$ image using IMAGR in AIPS by adding a zero spacing flux density of 2 Jy which was derived from multiple Gaussian fits to the visibility data, and determined that the total continuum flux density at $1.1 \mathrm{~mm}$ from the W3-SE core $\left(\sim 15^{\prime \prime} \times 15^{\prime \prime}\right)$ is $1.4 \pm 0.4 \mathrm{Jy}$. The large uncertainty in the flux density includes all possible error sources, dominated by the missing short spacings in the SMA observations.

\subsubsection{JCMT $\lambda 0.45 / \lambda 0.85 \mathrm{~mm}$}

Figures 1(c) and 1(d) show the continuum images observed with JCMT at $\lambda 0.85$ and $\lambda 0.45 \mathrm{~mm}$, respectively. With the angular resolutions $144^{\prime \prime} .5$ and 7.5 at $\lambda 0.85$ and $\lambda 0.45 \mathrm{~mm}$, the emission at submillimeter wavelengths from W3-SE has been slightly resolved, showing an extended, $\sim 1^{\prime}$ halo, which is better seen in the JCMT image at $\lambda 0.85 \mathrm{~mm}$ (Figure $1(\mathrm{c})$ ). An elongated core, $\sim 15^{\prime \prime}$, is clearly shown at $\lambda 0.45 \mathrm{~mm}$ in Figure $1(\mathrm{~d})$. The core-halo morphology of the continuum emission observed at submillimeter with the JCMT is consistent with CARMA and SMA observations at $\lambda 3.4$ and $\lambda 1.1 \mathrm{~mm}$, suggesting that the double compact continuum core (SMA-1 and SMA-2) revealed by the SMA is encompassed by a halo. Gaussian fits to the JCMT continuum images give peak positions $\left(-8.2 \pm 0.4,1^{\prime \prime} .2 \pm 0.6\right)$ and $(-8 . .8 \pm 0.4,0.5 \pm 0.4)$ for the $\lambda 0.45$ and $\lambda 0.85 \mathrm{~mm}$ emission, respectively. Within the JCMT beams, these two positions agree with the position of the continuum peak observed with CARMA at $\lambda 3.4 \mathrm{~mm}$ and the centroid position of the double components derived from the SMA observations at $\lambda 1.1 \mathrm{~mm}$.

From the JCMT images, integrating over emission region with signals greater than $5 \sigma$, we derived a total flux density $8 \pm 1$ and $75 \pm 8 \mathrm{Jy}$ at $\lambda 0.85$ and $0.45 \mathrm{~mm}$, respectively. All the measurements of flux densities at millimeter and submillimeter wavelengths are summarized in Table 2.

\subsubsection{MIPS and IRAC at Mid-infrared}

In the MIPS image at $24 \mu \mathrm{m}$ (Figure 2(a)), the direction of the elongation in the $24 \mu \mathrm{m}$ emission is in NW-SE (position angle, P.A. $\approx-25^{\circ}$ ). In comparison with the distribution of the emission at millimeter/submillimeter, we noticed that in the region located $\mathrm{E}$ of the SMA double sources there is a large excess emission at $24 \mu \mathrm{m}$, indicating a displacement between the hot and cold dust emission in the W3-SE core. 

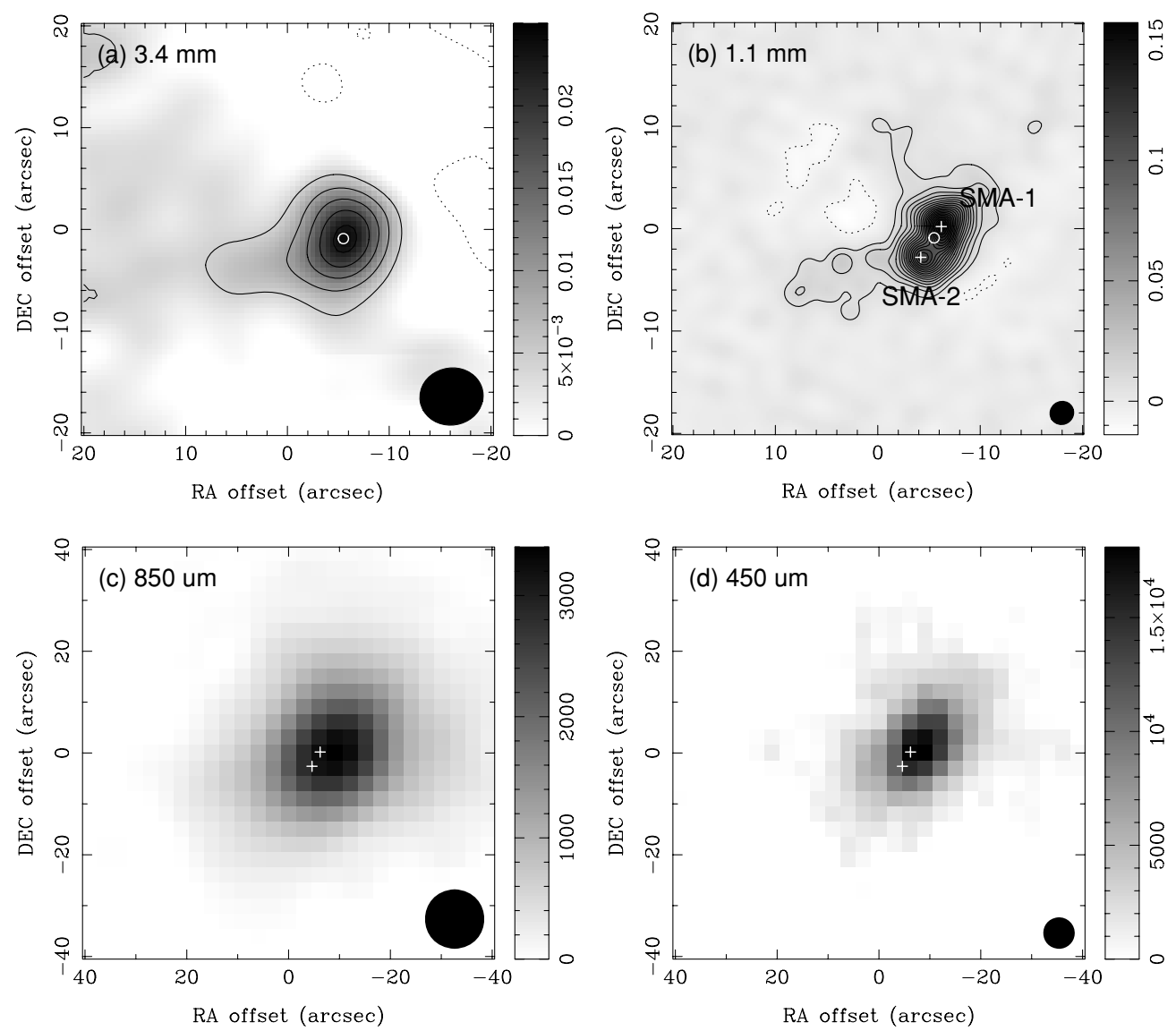

Figure 1. Dust emission of W3-SE. (a) The image of the continuum emission at $\lambda 3.4 \mathrm{~mm}$ made from the CARMA observations by combining two $500 \mathrm{MHz}$ bands. The contours are $3 \sigma \times(-1,1,2,3, \ldots)$ and $1 \sigma=0.0015 \mathrm{Jy} \mathrm{beam}^{-1}$. The FWHM beam size given at bottom-right is $6{ }^{\prime \prime} 2 \times 5^{\prime \prime} \cdot 6$, P.A. $=-81^{\circ}$. (b) The image of continuum emission at $1.1 \mathrm{~mm}$ based on the SMA observations in the compact array with two $2 \mathrm{GHz}$ side bands. The contours are $5 \sigma \times(-1,1,2,3, \ldots)$ and $1 \sigma=$ $0.0015 \mathrm{Jy}_{\text {beam }}{ }^{-1}$. The FWHM beam size is $2^{\prime \prime} .9 \times 2^{\prime \prime} .7$, P.A. $=-64^{\circ}$. The open circle in both (a) and (b) marks the peak position of the $3.4 \mathrm{~mm}$ emission. The images of continuum emission at $0.85 \mathrm{~mm}$ (c) and $0.45 \mathrm{~mm}$ (d) were made with the JCMT observations with the FWHM beam sizes of $144^{\prime \prime} 5$ and 7".5, respectively. The crosses in (b)-(d) mark the peak positions of the double sources (SMA-1 and 2). The units of the wedge in (a) and (b) are Jy beam ${ }^{-1} ; \mathrm{mJy} \mathrm{beam}^{-1}$ for (c) and (d).

Table 2

Measurements of the Continuum at Millimeter/Submillimeter

\begin{tabular}{|c|c|c|c|c|c|c|}
\hline \multirow{2}{*}{$\begin{array}{c}\lambda \\
(\mathrm{mm})\end{array}$} & \multirow[t]{2}{*}{ Telescope } & \multicolumn{4}{|c|}{ Gaussian Fitting Results } & \multirow{2}{*}{$\begin{array}{c}\mathrm{S}_{v} \\
(\mathrm{Jy})\end{array}$} \\
\hline & & $\operatorname{Peak}\left({ }^{\prime \prime}\right)$ & $I_{\text {peak }}\left(\mathrm{Jy}_{\mathrm{beam}}{ }^{-1}\right)$ & Sizes $\left({ }^{\prime \prime}\right)$ & P.A. $\left({ }^{\circ}\right)$ & \\
\hline 3.4 & CARMA & $-5.5,-0.9$ & $0.024 \pm 0.002$ & $7.9 \times 5.9$ & -51 & $0.04 \pm 0.01$ \\
\hline \multirow[t]{3}{*}{1.1} & SMA & $\ldots$ & $\ldots$ & $\ldots$ & $\ldots$ & $1.4 \pm 0.4$ \\
\hline & (SMA-1) & $-6.2,0.2$ & $0.21 \pm 0.01$ & $4.4 \times 3.0$ & 21 & $0.72 \pm 0.01$ \\
\hline & (SMA-2) & $-4.2,-2.8$ & $0.17 \pm 0.01$ & $3.5 \times 1.5$ & 76 & $0.35 \pm 0.01$ \\
\hline 0.85 & JCMT & $-8.2,1.2$ & $3.3 \pm 0.1$ & $25 \times 18$ & -48 & $8 \pm 1$ \\
\hline 0.45 & JCMT & $-8.8,0.5$ & $17.1 \pm 0.7$ & $18 \times 11$ & -41 & $75 \pm 8$ \\
\hline
\end{tabular}

Notes. Columns 1 and 2 give the wavelengths and telescopes that are used in the observations. Columns 3-6 are the results from Gaussian fitting to the sources, corresponding to peak positions, peak intensities, core sizes, and P.A.'s. Column 5 gives the total flux densities integrating the emission $>5 \sigma$ over the continuum core region of $15^{\prime \prime} \times 15^{\prime \prime}$ (except for SMA- 1 and SMA-2). The uncertainties are attributed mainly to the missing short spacing data for the interferometer observations and the uncertainty in determination of the boundary for the continuum core in the JCMT images. For SMA-1 and SMA-2, the total flux densities are determined from multiple Gaussian fitting to the $1.1 \mathrm{~mm}$ dust emission.

Based on the IRAC images, a group of discrete emission sources were revealed in the core of W3-SE (see the $3.6 \mu \mathrm{m}$ image in Figure 2(b)). By fitting PSF, three point sources were detected in the central core region of W3-SE. One of them has been reported in Ruch et al. (2007) as the IRAC point source 56 (hereafter IRAC 56), the only source that can be fitted as a point source in all of the four IRAC bands. The flux densities of IRAC 56 determined from this paper are in agreement with the results of Ruch et al. (2007). Located $\sim 4^{\prime \prime}$ NW to IRAC
56 , a weak point source (hereafter IRAC 56a) was detected at $4.5,5.8$, and $8.0 \mu \mathrm{m}$, corresponding to a weak emission feature at $3.6 \mu \mathrm{m}$ which failed to be fitted as a point source. The detection of this source is also in good agreement with the study of G. T. Ruch et al. (2009, private communication). This source appears to coincide with the core SMA-1, showing a large excess in the emission at the longer wavelengths (5.8 and $8.0 \mu \mathrm{m})$ as compared with that at 4.5 and $3.6 \mu \mathrm{m}$. The large excesses in emission at longer wavelengths suggest that 

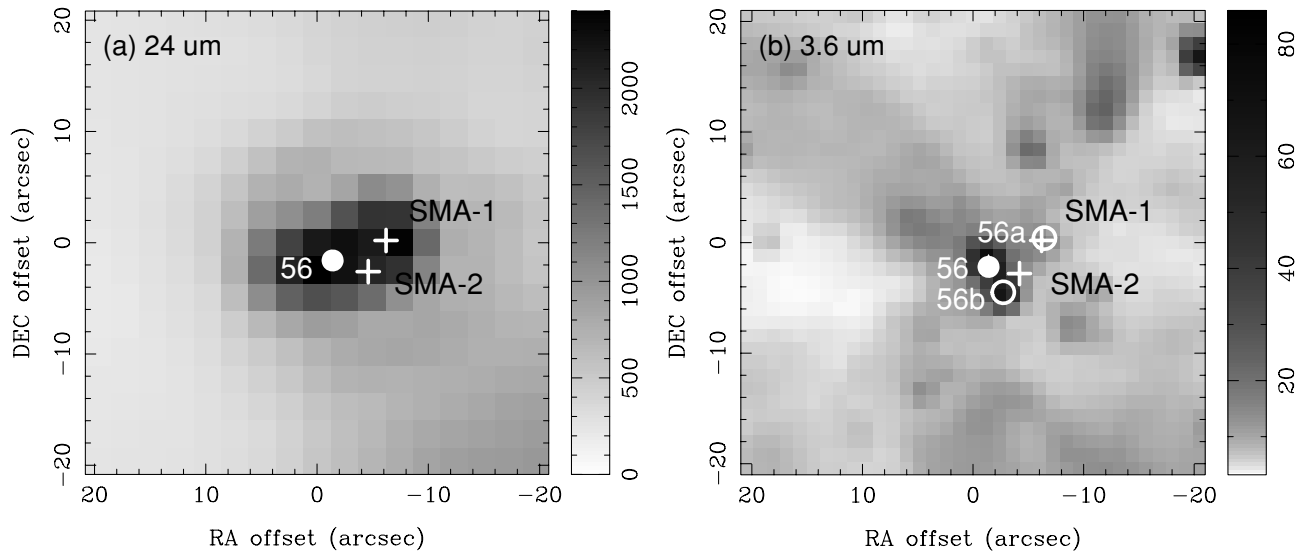

Figure 2. Spitzer images at (a) MIPS $24 \mu \mathrm{m}$ and (b) IRAC $3.6 \mu \mathrm{m}$. In the two images, the wedge scales the continuum emission in units of $10^{6} \mathrm{Jy} \mathrm{sr}^{-1}$, which is equivalent to $2.35 \times 10^{-5} \mathrm{Jy} \mathrm{arcsec}^{-2}$. The crosses in the two images indicate the peak positions of the double sources. The filled circle in (a) and (b) marks position of the mid-IR point source (IRAC 56) which has been identified by Ruch et al. (2007). The open circles in (b) indicate two additional sources (IRAC 56a and 56b) in the central core region of W3-SE.
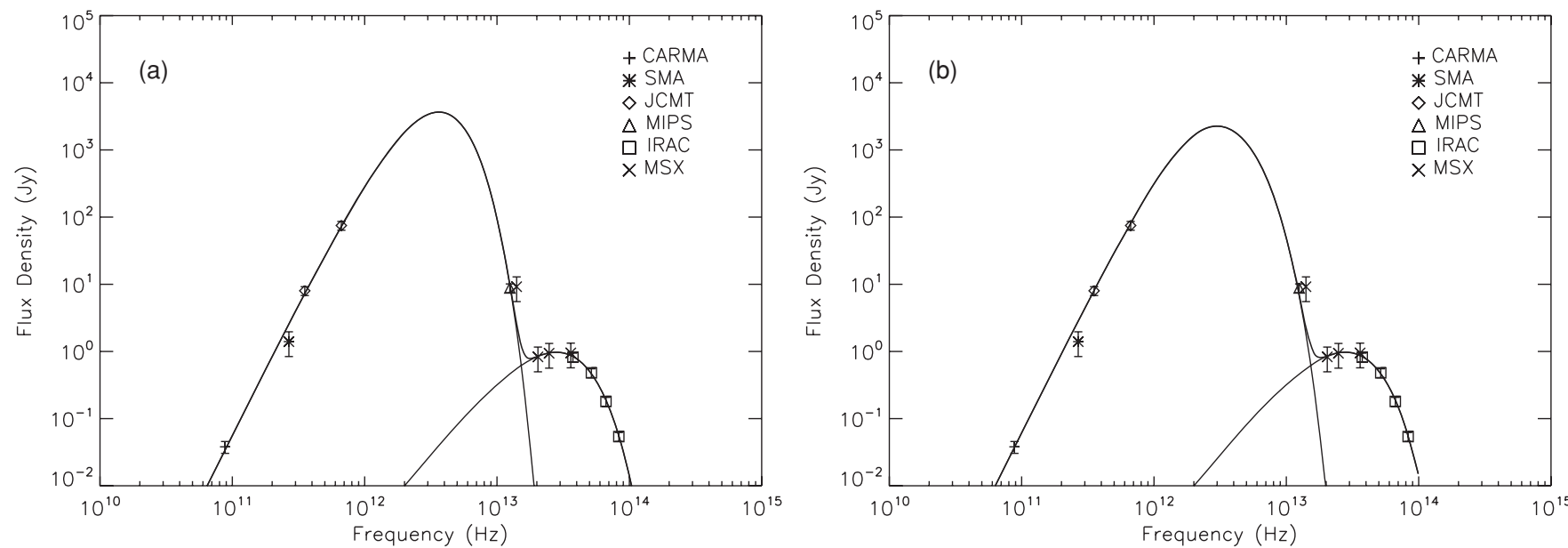

Figure 3. SED of the continuum emission from the W3-SE core determined from the observations with the CARMA, SMA, JCMT, MSX, MIPS, and IRAC. The legend (top-right) shows the symbols corresponding to each of the telescopes. The thick solid curve outlines the resulting SED fitting from two thermal dust components (indicated by the thin curves) determined from our model fitting. (a) The best (LSQ) fit to the flux density measurements of the SED assuming the optically thin dust emission from both cold and hot components. (b) A good fit to the SED considering the effect of self-absorption of the cold component with $\beta=2$ and assuming that the cold component does not overlap the hot dust emission region. The turnover frequency $v_{\tau=1}$ of the cold component is shifted by $\Delta v=80 \mathrm{GHz}$ to higher frequency as compared to that derived from the optically thin approach.

a protostar is probably embedded in SMA-1, and extinctions at shorter wavelengths become considerable. Located $\sim 2^{\prime \prime} \mathrm{SW}$ to IRAC 56 there appears to be an additional point source (hereafter IRAC 56b) detected at $4.5 \mu \mathrm{m}$, but we failed to fit it as a point source due to the contamination from the profile wing of the strong point source IRAC 56 and possible extended PAH or $\mathrm{H}_{2}$ emission. The nature of this source remains uncertain. In addition, we note that there appears no significant emission at the mid-IR wavelengths toward the cold dust core SMA-2.

Following the procedures of the aperture photometry described earlier, we determined the mid-IR flux densities from the core region of W3-SE. The total flux densities of W3-SE at mid-IR wavelengths from the IRAC and MIPS images, as well as the values from the MSX images, are summarized in Table 3.

\subsubsection{Dust Content and SED}

Figure 3 shows the spectral energy distribution (SED) of W3-SE covering the wavelengths from millimeter to mid-IR. We fit the SED with a thermal dust model to determine the physical parameters of the dust components in the core of W3SE. Given the dust temperature $T_{\mathrm{d}}$ and the total mass of gas
Table 3

The Continuum Flux Densities at Mid-IR

\begin{tabular}{lcc}
\hline \hline $\begin{array}{c}\lambda \\
(\mu \mathrm{m})\end{array}$ & Instrument & $\begin{array}{c}\mathrm{S}_{v} \\
(\mathrm{Jy})\end{array}$ \\
\hline 24 & MIPS & $8.8 \pm 1.3$ \\
21.3 & $M S X$ & $9.2 \pm 3.7$ \\
14.7 & $M S X$ & $0.83 \pm 0.33$ \\
12.1 & $M S X$ & $0.94 \pm 0.38$ \\
8.3 & $M S X$ & $0.95 \pm 0.38$ \\
8.0 & IRAC & $0.82 \pm 0.16$ \\
5.8 & IRAC & $0.48 \pm 0.10$ \\
4.5 & IRAC & $0.18 \pm 0.04$ \\
3.6 & IRAC & $0.054 \pm 0.011$ \\
\hline
\end{tabular}

Note. The observing wavelengths, the relevant instrument, and the continuum flux densities are given in Columns $1-3$, respectively.

and dust $M_{\text {total }}$, the continuum emission at the frequency $v$ from thermal dust in a molecular core subtending a solid angle $\Omega$ can be described as (Wilson et al. 2009)

$$
S_{v}=B_{v}\left(T_{\mathrm{d}}\right)\left(1-e^{-\tau_{v}}\right) \Omega,
$$


where

$$
\tau_{\nu}=M_{\text {total }} \kappa_{\nu 0}\left(\nu / \nu_{0}\right)^{\beta} / g D^{2} \Omega .
$$

In the optically thin case $\tau_{v}<1$, Equation (1) can be approximately expressed as

$$
S_{v} \approx \frac{M_{\mathrm{total}} \kappa_{v} B_{v}\left(T_{\mathrm{d}}\right)}{g D^{2}},
$$

where $\kappa_{v}$ is the dust opacity per unit dust mass, $g$ is the density ratio of $\mathrm{H}_{2}$ gas to dust, $D$ is the distance, and $B_{v}\left(T_{\mathrm{d}}\right)$ is the Planck function with a dust temperature of $T_{\mathrm{d}}$. Adopting $\kappa(300 \mathrm{GHz})=$ $1.4 \mathrm{~cm}^{2} \mathrm{~g}^{-1}$ as the reference value (Ossenkopf \& Henning 1994), we assumed a power law of $\kappa_{v}=\kappa_{0}\left(v / \nu_{0}\right)^{\beta}$ (Hildebrand 1983), where $\beta$ is the power-law index for dust opacity. In addition, we adopted $g=100$ and $D=2 \mathrm{kpc}$ in the following analysis. Thus, for each dust component, the thermal dust model has three free parameters $\left(M_{\mathrm{total}}, T_{\mathrm{d}}\right.$, and $\left.\beta\right)$.

Figure 3(a) shows the least square (LSQ) fitting to the SED with a total of 13 measurements from submillimeter to mid-IR on the assumption of optically thin dust emission. We found that a model with two thermal dust components appears to adequately fit the data. The six free physical parameters of the model can be determined by the LSQ fitting to the data. Our best fitting suggests that a cold, massive dust component accounts for the continuum emission at the millimeters/submillimeters as well as a large fraction of the continuum emission at $24 \mu \mathrm{m}$. A total mass of $M_{\text {total }}=70 \pm 7 M_{\odot}$, a dust temperature of $T_{\mathrm{d}}$ $=35 \pm 1 \mathrm{~K}$, and a dust opacity index of $\beta=2.0 \pm 0.1$ were derived from the fitting for this cold, massive dust component. In addition, the emission bump at mid-IR requires a secondary dust component with a small mass but a high temperature. Our fitting gives $T_{\mathrm{d}}=400_{-50}^{+70} \mathrm{~K}, \beta=0.5_{-0.5}^{+0.9}$, and $M_{\text {total }}<5 \times 10^{-4} M_{\odot}$ for the secondary component. We note that the uncertainties of the derived parameters for the secondary component are large due to the large uncertainties in the mid-IR measurements, especially the ones from $M S X$.

Although the optically thin approach is valid for the emission from the cold dust component at millimeter and submillimeter wavelengths and the emission from the hot component at mid-IR, the optical depth of the cold component may become significantly large at the wavelengths of mid-IR. On the assumption that the steep power law of the dust opacity $(\beta=2)$ holds up to the mid-IR, the mean optical depth of $\tau_{\nu} \sim 160$ at $\lambda 24 \mu \mathrm{m}$ for the cold dust in the region of the double source is inferred using the values of $T_{\mathrm{d}}$, and $M_{\text {total }}$ derived from the optically thin approach. Thus, the optical-depth effect cannot be ignored for the dust emission at the shorter wavelengths. Figure 3(b) shows a good fit to the observed SED data, considering selfabsorption in the cold dust with Equation (1) and assuming that cold component does not overlap the hot dust emission region. Consequently, in the self-absorption model the turnover frequency $v_{\tau=1}$, where $\tau(v)=1$, is shifted to the higher frequency of $1070 \mathrm{GHz}$ as compared with the value $\left(v_{\tau=1}=990 \mathrm{GHz}\right) \mathrm{de}$ rived in the optically thin approach. The self-absorption model gives a $15 \%$ decrease in $M_{\text {total }}$ and a $25 \%$ increase in $T_{\mathrm{d}}$, i.e., $T_{\mathrm{d}}=47 \mathrm{~K}$, and $M_{\text {total }}=60 M_{\odot}$, if the power-law index $\beta=2$ remains unchanged at mid-IR. In the case that the power-law of the dust opacity becomes flat, e.g., $\beta=0.5$ at the frequencies above $1000 \mathrm{GHz}$, then $\tau_{v} \sim 3$ at $\lambda 24 \mu \mathrm{m}$ and the optical depth effect at mid-IR becomes not so critical. In any case, the uncertainties in the determination of $T_{\mathrm{d}}$ and $M_{\mathrm{total}}$ appears to be mainly due to the uncertainty in the dependence of the opacity on frequency at mid-IR. Thus, we take the mean values of the physical parameters derived from the two extreme cases, namely $T_{\mathrm{d}}=41 \pm 6 \mathrm{~K}$ and $M_{\text {total }}=65 \pm 10 M_{\odot}$. Our result in dust mass is in good agreement with that of Zinchenko et al. (2009), which suggests a total mass of $74 M_{\odot}$ derived from dust continuum for W3-SE and a virial mass of $90 M_{\odot}$.

On the other hand, we can estimate the extinction from the cold dust component at mid-IR wavelengths according to recent studies of the mid-IR extinction law. Based on the highresolution observations with the SMA at $\lambda 1.1 \mathrm{~mm}$, most of the cold dust (over $\sim 85 \%$ ) is concentrated in the two compact millimeter/submillimeter sources (SMA-1 and 2), the two possible protostellar cores. Assuming an even distribution of the dust over the sizes of the two cores (see Column 5 of Table 2), the average column density of the $\mathrm{H}_{2}$ in the cores would be $1.5 \times 10^{24} \mathrm{~cm}^{-2}$. On the basis of the equation $\left[N(\mathrm{HI})+2 N\left(\mathrm{H}_{2}\right)\right] /$ $A_{K}=1.67 \times 10^{22} \mathrm{~cm}^{-2} \mathrm{mag}^{-1}$ from Lada et al. (2009), such a column density is equivalent to an extinction $A_{K}=\sim 200 \mathrm{mag}$ at $K$ band. Assuming a value of 0.5 for both $A_{\mathrm{IRAC}} / A_{K s}$ and $A_{24} / A_{K s}$ based on recent studies on the mid-IR extinction law (e.g., Flaherty et al. 2007), the corresponding extinctions at the IRAC bands and the $\lambda 24 \mu \mathrm{m}$ would be $\sim 100 \mathrm{mag}$. Obviously the light from the sources within the two cold dust cores is severely obscured at mid-IR wavelengths. The residual of the cold dust emission $(\sim 15 \%$, or $0.2 \mathrm{Jy}$ at $\lambda 1.1 \mathrm{~mm})$ appears to be distributed outside the two compact cores, corresponding to a mass of $\sim 10 M_{\odot}$. On the assumption of an even distribution of this extended cold dust component over a region of $0.15 \mathrm{pc} \times$ $0.15 \mathrm{pc}\left(15^{\prime \prime} \times 15^{\prime \prime}\right)$, the column density $N\left(\mathrm{H}_{2}\right)$ is about $2.5 \times$ $10^{22} \mathrm{~cm}^{-2}$, corresponding to an extinction of $\sim 3 \mathrm{mag}$ at $K$ band, or $\sim 1.5 \mathrm{mag}$ at the IRAC bands and $\lambda 24 \mu \mathrm{m}$. Considering the observed filamentary structure of the cold dust in the SMA and CARMA observations, the extinction of the extended cold dust distribution to the mid-IR emission from W3-SE varies around 1 mag. This extinction may have some effects on obscuring the emission from the hot dust component depending on the relative distribution of the extended cold dust with respect to the hot dust component.

Nevertheless, our fitting to the observed SED of W3-SE suggest the existence of a dominant cold dust component with a dust temperature of $41 \pm 6 \mathrm{~K}$ and a total mass of $65 \pm 10 M_{\odot}$. A secondary dust component which has a little mass but high dust temperature is necessary to account for the observed emission at IRAC wavelengths, which is likely heated by the nearby stellar objects (e.g., IRAC 56). The suggested heating process appears to be also consistent with the structure of the mid-IR emission characterized as several discrete compact components as discussed in the previous section (also see Figure 2).

\subsection{Molecular Lines}

\subsubsection{Non-detection of $\mathrm{SiO}$ (2-1) Line}

On the basis of CARMA observations of $\mathrm{SiO}(2-1)$ line at $86.243 \mathrm{GHz}$, we did not detect $\mathrm{SiO}$ emission at a $3 \sigma$ level $\left(\sim 0.3 \mathrm{Jy}\right.$ beam $\left.^{-1}\right)$ with a channel width of $0.42 \mathrm{~km} \mathrm{~s}^{-1}$. The $\mathrm{SiO}(2-1)$ line is not a good tracer for the outflows in W3-SE.

\subsubsection{Distribution of the $\mathrm{HCO}^{+}(1-0) \mathrm{Gas}$}

Figure 4 shows the channel maps of $\mathrm{HCO}^{+}(1-0)$ from W3-SE with the CARMA observations, revealing complex structures in kinematics and spatial distribution. Strong line emissions are present in the velocity range from -43.9 to $-33.2 \mathrm{~km} \mathrm{~s}^{-1}$ (above $3 \sigma$ ), with a gap between -38.6 and $-37.3 \mathrm{~km} \mathrm{~s}^{-1}$. The emission blueshifted with respect to the gap is stronger 


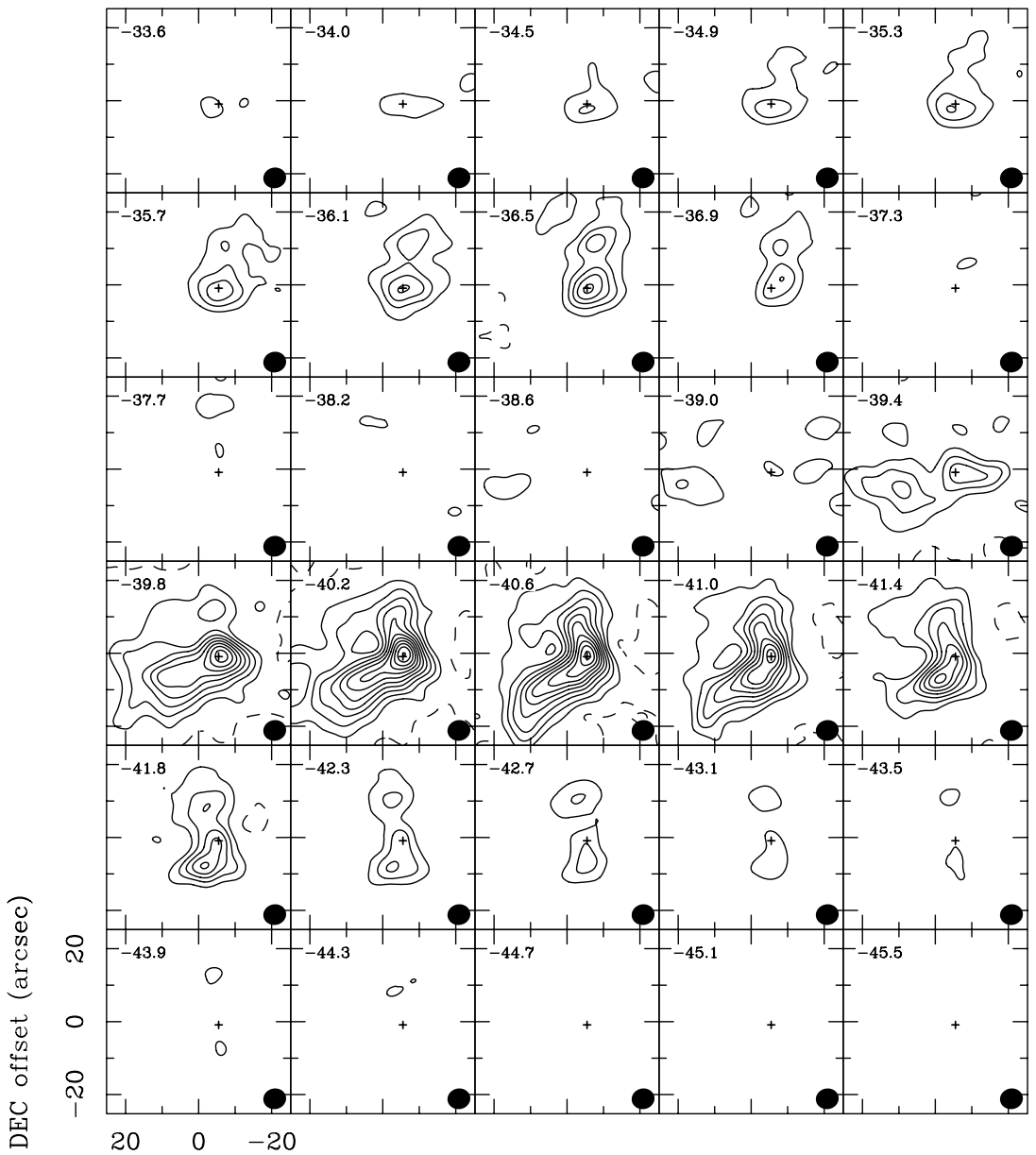

RA offset $(\operatorname{arcsec})$

Figure 4. Channel maps of the $\mathrm{HCO}^{+}(1-0)$ line observed with CARMA. The channel separation is $0.42 \mathrm{~km} \mathrm{~s}^{-1}$. The contours are $4 \sigma \times(-1,1,2,3, \ldots)$ and $1 \sigma=$ $0.1 \mathrm{Jy}_{\text {beam }}^{-1}$. The LSR velocity is labeled at top-right on each channel map. The FWHM beam (bottom-right) is $6{ }^{\prime \prime} 3 \times 5^{\prime \prime} \cdot 7$, P.A. $=-79^{\circ}$.

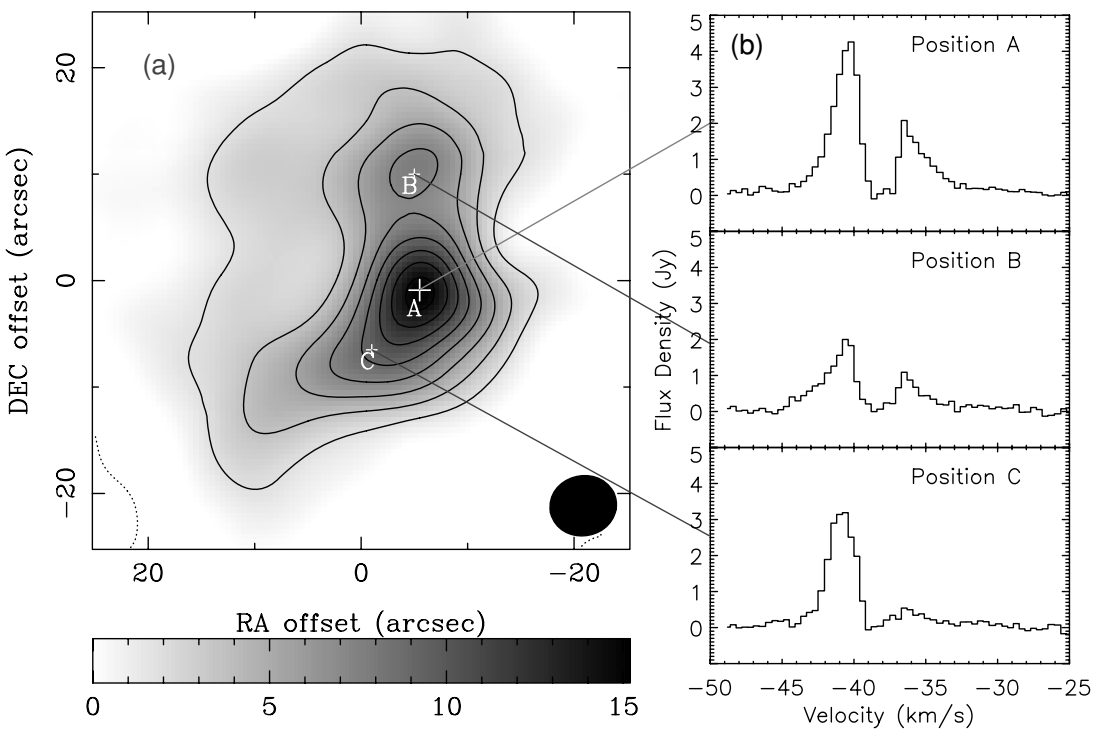

Figure 5. (a) Integrated intensity map of $\mathrm{HCO}^{+}(1-0)$ from the CARMA observations. The contours are $3 \sigma \times(-1,1,2,3, \ldots)$ and $1 \sigma=0.6 \mathrm{Jy}$ beam ${ }^{-1} \mathrm{~km} \mathrm{~s}^{-1}$. The FWHM beam size is $6^{\prime \prime} 3 \times 5^{\prime \prime} .7$, P.A. $=-81^{\circ}$, shown at bottom-right. The cross marks the $\lambda 3.4 \mathrm{~mm}$ continuum peak. (b) The spectra toward positions A, B, and C marked in Figure 5(a).

and more extended than the redshifted gas. Figure 5(a) shows the $\mathrm{HCO}^{+}(1-0)$ line intensity image integrated over all the line channels, delineating an arc-like morphology in the distribution of molecular gas. Hereafter, we denote the main component with the maximum line intensity as A, the northern and SE lobes as $\mathrm{B}$ and $\mathrm{C}$, respectively. The main component $\mathrm{A}$ is located at the 

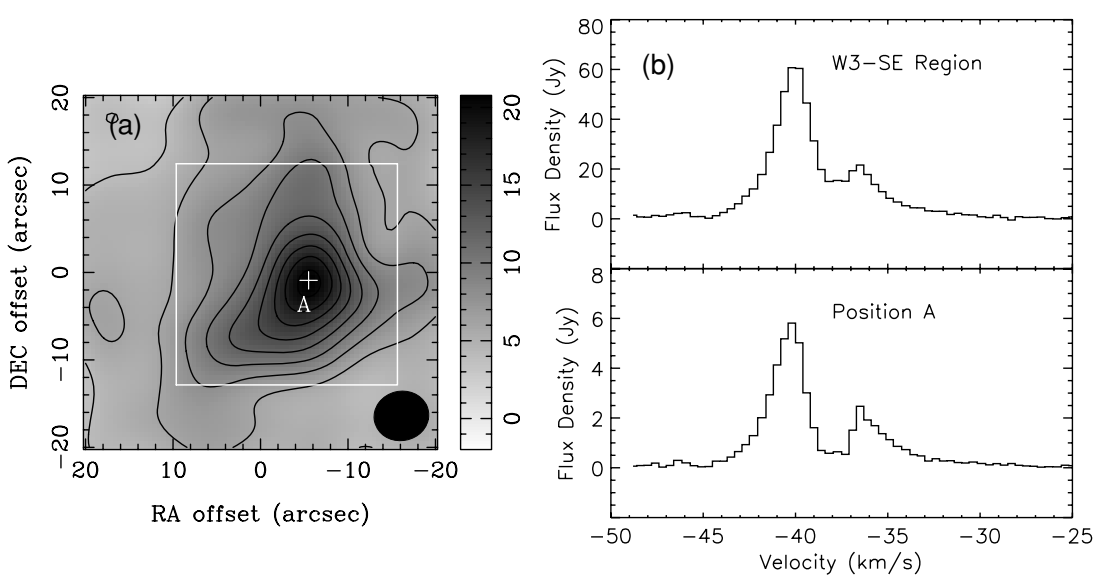

Figure 6. (a) Integrated intensity image of the $\mathrm{HCO}^{+}(1-0)$ line emission constructed by combining the CARMA data with the IRAM $30 \mathrm{~m}$ data. The beam size of 6!' $3 \times 55^{\prime \prime} 7$, P.A. $=-81^{\circ}$ is the same as the one of CARMA image (Figure 5(a)). The boundary effect has been discussed in the text. (b) The top spectrum is the integrated $\mathrm{HCO}^{+}(1-0)$ line emission within the $25^{\prime \prime} \times 25^{\prime \prime}$ square as delineated. The spectrum toward the peak position A is shown at the bottom.

position $\left(-5^{\prime \prime} 4,-1\right.$ '.5) offset from the reference center, which coincides with the continuum peak position at $\lambda 3.4 \mathrm{~mm}$ and the centroid position of the SMA double source. The spectra derived from the three positions (A, B, and C) are shown in Figure 5(b). The spectrum at A clearly shows a double-peak line profile; one is at $-40.0 \mathrm{~km} \mathrm{~s}^{-1}$ (or the "blue peak" hereafter) and the other at $-36.0 \mathrm{~km} \mathrm{~s}^{-1}$ (or "red peak" hereafter). A spectral dip to $\sim 0 \mathrm{Jy}$ between the blue and red peaks is shown, which is observed as an empty gap between -37.3 and $-38.6 \mathrm{~km} \mathrm{~s}^{-1}$ in the channel maps (Figure 4). In the velocity range from -34.9 to -36.9 $\mathrm{km} \mathrm{s}^{-1}$, the redshifted emission peak appears to coincide near the position A with the blueshifted emission peak in the velocity range from -39.4 to $-40.6 \mathrm{~km} \mathrm{~s}^{-1}$, suggesting that the red and blueshifted gas components originate from a common core. At position $\mathrm{A}$, the line peak intensities are 4.3 and $2.1 \mathrm{Jy}^{\mathrm{J}}$ beam $^{-1}$ for the red and blueshifted gas in W3-SE, respectively.

Position B is located at $\left(-5^{\prime \prime} .1,99^{\prime \prime}\right), \mathrm{N}$ of the main core $\mathrm{A}$, appeared to be a sub-core. At position B the line emission is much weaker than that at position A, but the line intensity ratio of the blue and red peaks is $\sim 2$, similar to that observed from position A. Toward position C $\left(-1{ }^{\prime \prime} 1,-6.6\right)$, the spectrum is dominated by the blueshifted line component, while the redshifted line emission appears to be very weak.

Besides the three relatively compact structures, there appears to be an extended halo, $\sim 40^{\prime \prime}$ in W3-SE. The total $\mathrm{HCO}^{+}(1-0)$ flux by integrating the line emission above $3 \sigma$ from the entire W3-SE region is $124 \mathrm{Jy} \mathrm{km} \mathrm{s}^{-1}$, which underestimates the true flux because of missing short spacing. The shortest projected baseline of the CARMA data is $2.7 \mathrm{k} \lambda$, suggesting missing information on the source structure with a size larger than $\sim 76^{\prime \prime}$. We combined the CARMA data with the IRAM $30 \mathrm{~m}$ data to assess the effects due to missing short spacing (Section 2.2).

Figure 6(a) shows the image constructed from combined CARMA and IRAM $30 \mathrm{~m}$ data with the same beam size $\left(6.3 \times 5.7\right.$, P.A. $\left.=-81^{\circ}\right)$ as that of the CARMA image (Figure 5(a)). The combined image shows better the emission from the extended halo but trades off the detailed structures. The total line flux of $260 \mathrm{Jy} \mathrm{km} \mathrm{s}^{-1}$ derived from the combined image is about twice as large as that derived from the CARMA data alone. Toward the peak position A, the spectrum shows that the intensity peak from the combined data is $\sim 25 \%$ higher than that of the CARMA data while the intensity at the spectral dip increases to $0.5 \mathrm{Jy}(5 \sigma)$ in the combined image from $\sim 0 \mathrm{Jy}$ of the CARMA image.

\subsubsection{Outflows}

The broad wings of the line profiles in the $\mathrm{HCO}^{+}(1-0)$ spectra suggest that outflow(s) is (are) active in this molecular cloud. We imaged the high-velocity portions of the outflow(s) by integrating the blue spectral wings from -41.4 to $-46.7 \mathrm{~km} \mathrm{~s}^{-1}$ and the red spectral wings from -29.6 to $-36.5 \mathrm{~km} \mathrm{~s}^{-1}$. Figure 7(a) shows the blue- and redshifted gaseous components in blue and red contours, respectively. It appears that the redshifted outflow lobe is located NW of the continuum core, while the blueshifted outflow lobe shows two components located SE (C) and N (B) of the continuum core, respectively. The complex outflow structure implies that there might be more than one outflow in W3-SE, and/or the direction of the outflow is close to the line of sight. Cutting along a direction from NW to SE (marked as the straight line with P.A. $=120^{\circ}$ in Figure 7(a)), we constructed a position-velocity (PV) diagram, showing a kinematic structure that appears to be consistent with a decelerated outflow (Qin et al. 2008). The PV diagram shows only the high velocity components that originate from the major core A. However, the major axis of the large-scale outflow is poorly determined due to the poor collimation of red- and blueshifted emission.

Line emission feature B mimics an independent molecular core based on the overlapping blue- and redshifted outflows shown in Figure 7(a) although we do not have further evidence either supporting or ruling out the possibility of it being a low-mass star-forming core. Feature B could also be produced from possible multiple outflows originated from the major core A. Line emission feature $\mathrm{C}$ appears to be dominated by a blueshifted outflow lobe originated from the major core A. To clarify the multiplicity of the outflows in W3-SE, higherresolution observations are necessary.

\subsubsection{Possible Infall Signature}

Blueshifted line profiles were discovered by Wu et al. (2007) in $\mathrm{HCO}^{+}(1-0)$ and other optically thick lines, which serve as an infall signature (Mardones et al. 1997). Figure 5(b) shows the spectra at three positions of the components A, B, and C. All the spectral profiles toward the three regions appear to be characterized by an asymmetric line profile with a dominated blueshifted peak and a weaker redshifted peak. However, $\mathrm{Wu}$ et al. (2007) also noticed the existence of significant line wings in $\mathrm{HCO}^{+}$lines from their IRAM $30 \mathrm{~m}$ observation. From our 

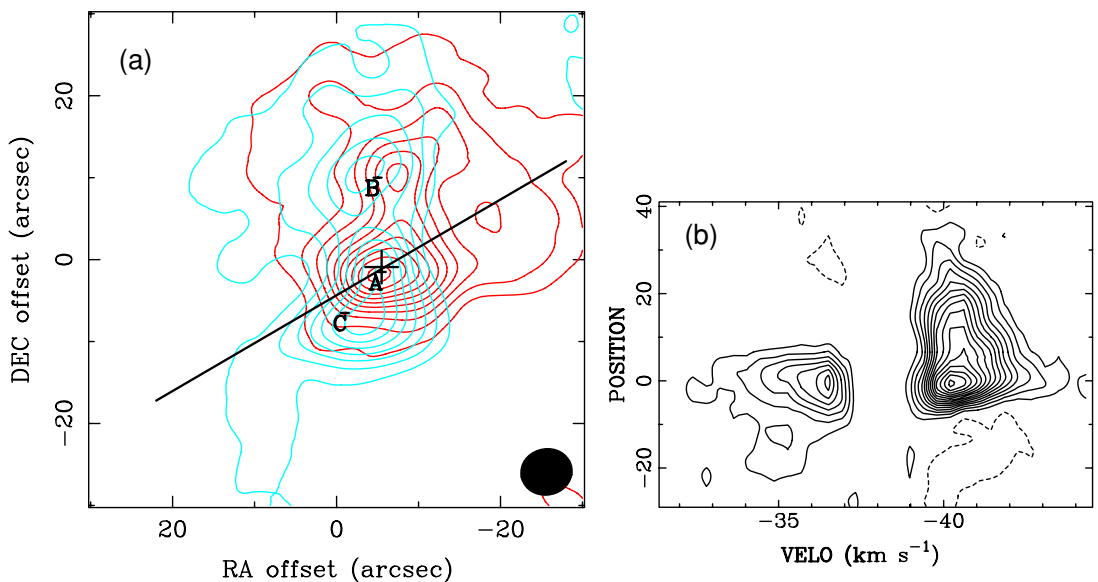

Figure 7. Integrated intensity maps for the blue and red wings based on CARMA data. The contours are $5 \sigma \times(1,2,3, \ldots)$ and $1 \sigma=0.12 \mathrm{Jy} \mathrm{beam}^{-1} \mathrm{~km} \mathrm{~s}^{-1}$. The FWHM beam size is $6.3 \times 5^{\prime \prime} .7$, P.A. $=-81^{\circ}$. The straight line denotes the direction of the cut for the PV diagram in (b). (b) The PV diagram along the cut indicated in Figure 7(a). The vertical axis is the offset relative to the position A, in units of arcsec, positive to SE. The contours are $5 \sigma \times(1,2,3, \ldots)$ and $1 \sigma=0.08 \mathrm{Jy}$ beam ${ }^{-1}$.

CARMA observations, the observed asymmetric double-peak profiles in spectra toward B and C appear to be due to outflow. We have shown good evidence for star formation activities occurring in W3-SE and kinematics of the molecular gas in the central $50^{\prime \prime}$ region which is likely dominated by outflows. We made an attempt to investigate the possible contribution from infall in the line profile of the spectrum toward A, the dust core, with a model including both infall and outflow.

In order to quantitatively differentiate the contributions from different dynamical processes in the resulting spectral profile, we utilize the infall model of Myers et al. (1996) to fit the spectral line profile. Four parameters: peak optical depths $\left(\tau_{0}\right)$, kinetic temperature $\left(T_{\mathrm{k}}\right)$, velocity dispersion $(\sigma)$, and the characteristic velocity $\left(V_{\text {in }}\right)$ with respect to the system velocity of $\left(V_{\text {sys }}\right)$, are used in a simple model to characterize two layers of infall gas. We found that, indeed, it is difficult to fit the observed spectral profile using a model with only infall components because of the broad blue- and redshifted wings producing sizable residuals in the fitting.

Thus, we introduced an additional two-layer outflow component with four more characteristic parameters, namely, brightness temperature $T_{\mathrm{b} \text {,out }}$, optical depth $\left(\tau_{0, \text { out }}\right)$, characteristic velocity $\left(V_{\text {out }}\right)$ with respect to the system, and the velocity dispersion $\left(\sigma_{\text {out }}\right)$ in the Gaussian profile. Similar approach has been considered in modeling the infall motions in NGC 1333 IRAS 4 (Attard et al. 2009). Here, Figure 8 shows the result of fitting the observed spectral profile with the modified infall model. Assuming $T_{\mathrm{b} \text {,out }}=50 \mathrm{~K}$ and $V_{\text {out }}=2 \mathrm{~km} \mathrm{~s}^{-1}$, the best fitting gives $\tau_{0}=4.1 \pm 0.4, T_{k}=32 \pm 5 \mathrm{~K}, \sigma=0.64 \pm 0.04 \mathrm{~km} \mathrm{~s}^{-1}$, $V_{\text {in }}=0.86 \pm 0.06 \mathrm{~km} \mathrm{~s}^{-1}, V_{\text {sys }}=-38.6 \pm 0.1 \mathrm{~km} \mathrm{~s}^{-1}$ for the infall component (the dashed profile in Figure 8) and $\tau_{0, \text { out }}=0.2 \pm 0.02$ and $\sigma_{\text {out }}=1.7 \pm 0.1 \mathrm{~km} \mathrm{~s}^{-1}$ for the outflow (dotted profile). The spectral dip cannot be produced with a simple superimposition of the double-Gaussian outflow profile (inset (a) in Figure 8) to the infall profile (inset (b) in Figure 8). The spectral dip seen in the resulting line profile is due to the absorption of the cold infall gas located in front of the outflow. After solving the radiative transfer equations, the final resulting profile of the model fits the data reasonably well.

The result for the outflow component is not unique. Other combinations of the three parameters $T_{\mathrm{b} \text {,out }}, V_{\text {out }}$, and $\tau_{0 \text {,out }}$ are also possible to produce a good fit to the observed line profile. In addition to the second kinematic component due to

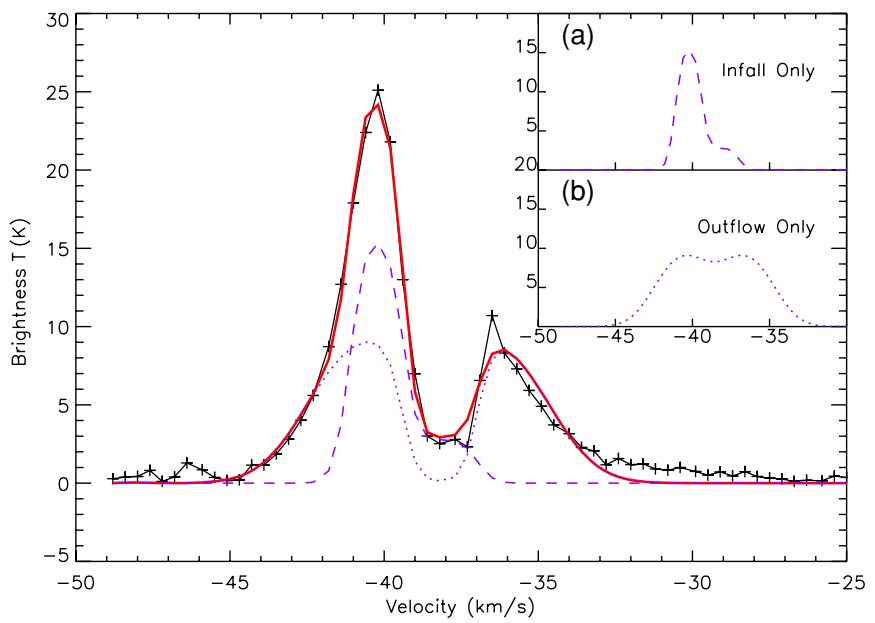

Figure 8. Observed line profile (crosses connected with a black curve) fitted with a two-layer infall model including blue- and redshifted outflow components. The solid curve (red) shows the resulting profile from the infall (dashed curve) and outflow (dotted curve) components. The insets at the top-right show the two spectra produced separately with infall alone (upper) and outflow alone (lower) using the same parameters derived from the overall spectra fitting.

outflow, any other kinematic components within the CARMA beam $\left(\sim 6^{\prime \prime}\right)$ characterized with two Gaussians separated in velocity, such as two sub-cores orbiting around the mass center, can be incorporated into the model in a way similar to that of the outflow, i.e., the dynamics in the central $6^{\prime \prime}$ may be equally important in the production of the observed spectral profile. Furthermore, we also note that the observed asymmetric line profile can be produced by an outflow alone if an unequal optical depth is used for red- and blueshifted components in the outflow. Although the modeling is suggestive, we need improved observations with high-angular resolution to differentiate between the dynamical processes.

The $V_{\text {sys }}=-38.6 \mathrm{~km} \mathrm{~s}^{-1}$ derived from the model fitting differs from the mean value $\left(-38.9 \mathrm{~km} \mathrm{~s}^{-1}\right)$ of the peak velocities from the single-peak optically thin lines such as $\mathrm{C}^{18} \mathrm{O}$ (1-0) observed with single dish (Wu et al. 2007). Such a mean peak velocity derived from observations of optically thin molecular lines is closer to the systematic velocity of the cores. The small discrepancy between the values of $V_{\text {sys }}$ predicted by our model and derived from observations might be due to the assumption of equal optical depth in the blue- 
and redshifted outflows used in the model. Furthermore, the absorption dip in the observed $\mathrm{HCO}^{+}(1-0)$ spectrum is centered at $\sim-38.0 \mathrm{~km} \mathrm{~s}^{-1}$, redshifted relative to the systematic velocity $\left(-38.9 \mathrm{~km} \mathrm{~s}^{-1}\right)$ by $0.9 \mathrm{~km} \mathrm{~s}^{-1}$, which is in good agreement with the value of $V_{\text {in }}$ derived from the model fitting.

With the caveats discussed above, we can calculate the kinematic infall rate $d M / d t=4 \pi r^{2} m_{\mathrm{H}} \mu n_{\mathrm{nr}} V_{\text {in }}$ assuming spherical geometry. Given a radius $r=6 \times 10^{3} \mathrm{AU}$ for the main core A, a critical density of $\mathrm{HCO}^{+}(1-0) n_{\mathrm{nr}}=1.8 \times 10^{5} \mathrm{~cm}^{-3}$ (Evans 1999) and a mean molecular weight $\mu=2.35$, an infall rate $d M / d t=9.7 \times 10^{-5} M_{\odot} \mathrm{yr}^{-1}$ is inferred. This infall rate appears to be consistent with the global gravitational rate $a^{3} / G=6.8 \times 10^{-5} M_{\odot} \mathrm{yr}^{-1}$ (Shu 1977) assuming that the isothermal sound speed $a$ equals the resulting velocity dispersion $\sigma\left(0.6 \mathrm{~km} \mathrm{~s}^{-1}\right)$. Indeed, the sound speed of ideal $\mathrm{H}_{2}$ gas at $T=40 \mathrm{~K}$ is $\sqrt{1.4 k T / m_{\mathrm{H}_{2}}}=0.5 \mathrm{~km} \mathrm{~s}^{-1}$, consistent with the value of the fitted $\sigma$.

Alternatively, rotation along with other dynamical process and the depletion of $\mathrm{HCO}^{+}$in the range of velocity close to the systematical velocity might be also responsible to the observed asymmetric double-peak line profile (Pavlyuchenkov et al. 2008). Especially, the velocity gradient as indicated in the $\mathrm{PV}$ diagram of the optically thin $\mathrm{C}^{18} \mathrm{O}(1-0)$ line (see Wu et al. 2007) suggests that a rotation may indeed play an important role in formation of the observed line profile. In addition, although core A has been resolved into two continuum cores in the SMA observations, this multiplicity does not account for the observed double-peak line profile since further molecularline observations with higher angular resolution show that the double-peak line profile exists in both of the cores (Zhu et al. 2010, in preparation).

\section{DISCUSSION}

\subsection{The Dynamical Age and Mass of the Outflows}

The dynamical age of the outflow can be inferred from the spatial extension and the mean velocity of the outflow. In the case of W3-SE, the maximum relative velocities $(>5 \sigma)$ of the blueand redshifted outflow lobes from the core A are $\sim 6 \mathrm{~km} \mathrm{~s}^{-1}$ (see the PV diagram in Figure 7(b)) with respect to the systematic velocity of $-38.9 \mathrm{~km} \mathrm{~s}^{-1}$ (Wu et al. 2007). Assuming a size $\sim 20^{\prime \prime}\left(\sim 4 \times 10^{4} \mathrm{AU}\right)$ for each lobe, the dynamical age of the outflow is $\sim 3 \times 10^{4}$ yr. Since the major axis of the outflow is poorly determined and its direction might be close to the line of sight, the estimated dynamical age corresponds to a low limit. Given the non-detection of radio continuum, both the typical outflow velocity and the dynamical age indicate that W3-SE probably hosts intermediate- and/or low-mass stars.

We calculated the mass associated with the main outflow in W3-SE using the formula for column density in the case of a linear, rigid rotor molecule on the assumption of local thermodynamic equilibrium (Scoville et al. 1986). For $\mathrm{HCO}^{+}(1-0)$, the total column density at all energy levels can be estimated following the equation:

$$
\begin{aligned}
N\left(\mathrm{HCO}^{+}\right)= & \frac{3 k}{8 \pi^{3} B \mu_{d}^{2}}\left(T_{\mathrm{ex}}+\frac{h B}{3 k}\right) \\
& \times\left(1-e^{-\frac{h v}{k T_{\mathrm{ex}}}}\right)^{-1} \int \tau_{v} d v,
\end{aligned}
$$

where we adopted the rotational constant $B=44.594 \mathrm{GHz}$ and the permanent electric dipole moment $\mu_{\mathrm{d}}=3.30$ debye (Woods et al. 1975). Assuming optically thin gas and excitation temperature of $T_{\mathrm{ex}}=40 \mathrm{~K}$, the optical depth $\tau$ is proportional to the brightness temperature $T_{B}$ (Wilson et al. 2009):

$$
\tau=\frac{k T_{B}}{h v}\left(\frac{1}{e^{h v / k T_{\text {ex }}}-1}-\frac{1}{e^{h v / k T_{b g}}-1}\right)^{-1} .
$$

The outflow mass can be calculated from Equation (3) in the case of optically thin emission, which valid for the outflow gas in the high-velocity wings,

$$
\begin{aligned}
M\left(\mathrm{H}_{2}\right)= & X_{\mathrm{HCO}^{+}}^{-1} m\left(\mathrm{H}_{2}\right) D^{2} \\
& \times \int N\left(\mathrm{HCO}^{+}\right) d \Omega,
\end{aligned}
$$

where we adopted a fractional abundance $\mathrm{X}_{\mathrm{HCO}^{+}}=7.6 \times$ $10^{-9}$ for W3-SE (Zinchenko et al. 2009) and a distance $D=$ $2 \mathrm{kpc}$. Then, we determined the velocity-integrated brightness temperature averaged over the emission region $\Omega \sim$ $50^{\prime \prime} \times 50^{\prime \prime}, \int\left\langle T_{B}\right\rangle_{\Omega} d v=1.2 \mathrm{~K} \mathrm{~km} \mathrm{~s}^{-1}$ between -46.7 and $-41.8 \mathrm{~km} \mathrm{~s}^{-1}$, and $\int\left\langle T_{B}\right\rangle_{\Omega} d v=1.4 \mathrm{~K} \mathrm{~km} \mathrm{~s}^{-1}$ between -36.5 and $-31.6 \mathrm{~km} \mathrm{~s}^{-1}$. The corresponding masses are 2.5 and $2.8 M_{\odot}$ for the blue- and redshifted outflow lobes, respectively. Consequently, with the maximum relative velocities of the outflow lobes $\left(\sim 6 \mathrm{~km} \mathrm{~s}^{-1}\right)$ and the dynamic age $\left(\sim 3 \times 10^{4} \mathrm{yr}\right)$, the momentum rates for both the blue and red lobes are $\sim 5 \times$ $10^{-4} M_{\odot} \mathrm{km} \mathrm{s}^{-1} \mathrm{yr}^{-1}$, corresponding to mechanical luminosities of $0.2 L_{\odot}$. The inferred outflow momentum rates and mechanical luminosities appear to be consistent with B-type stars in W3-SE (Arce et al. 2007).

\subsection{A Group of Young Stars/Protostars in W3-SE}

Observations at both millimeter and submillimeter wavelengths, together with those at mid-IR suggest that the main molecular core A hosts a group of young stars and/or protostars. The millimeter and submillimeter observations suggest that the cold dust is distributed in an elongated region from SE to NW (Figure 2) with two dust condensations separated by 4 ". The non-detection of radio continuum at $\lambda 6 \mathrm{~cm}$ (Tieftrunk et al. 1998 ) indicates that no OB stars have been found in W3-SE yet although we cannot rule out the possibility that the two sub-cores in the molecular core A (SMA-1 and SMA-2) will continue to accrete from the molecular gas reservoir of $\sim 65 M_{\odot}$ and form a massive star. The four IRAC images show a small group of discrete sources in the dust core. One (IRAC 56) of them has been identified by Ruch et al. (2007) as a point source, suggesting an association with a YSO earlier than Class I. This source appears to be one of the youngest objects in the entire W3 cluster as indicated in the color-color diagram of Ruch et al. (2007). The weak point-like source IRAC 56a might be associated with a protostar embedded in the dust core SMA-1.

The positions of the emission peaks in the millimeter/ submillimeter continuum and lines appear to be displaced significantly from the brightest mid-IR sources in this region. The majority of the mid-IR emission arises from the region located east of the molecular core (see Figure 2). The displacement likely indicates that the hot and cold dust components which are inferred from fitting the SED are also spatially separated in the W3-SE region. The spatial separation between the hot and cold dust might be explained by sequential star formation in W3-SE. Namely, stars formed first in the region east to the main molecular core A in W3-SE, where the dust/molecular components might have been substantially dissociated or destroyed by the stars. The activity of forming new stars is now taking place in A. 
The activity of star formation appears to propagate from $\mathrm{E}$ to $\mathrm{W}$ in W3-SE. Alternatively, the displacement of the emission peak position at mid-IR from that of millimeter/submillimeter could be due to possible strong mid-IR extinction in the main molecular core A. The scenario of sequential activities in star formation needs to be verified by further improved mid-IR observations with corrections for dust extinction.

\section{SUMMARY AND CONCLUSIONS}

We observed W3-SE with CARMA and SMA at $\lambda 3.4$ and $\lambda 1.1 \mathrm{~mm}$. Using the continuum flux densities determined with CARMA, SMA, and JCMT together with measurements at mid-IR wavelengths with Spitzer and MSX, we constructed the SED for the W3-SE core. The SED can be fitted with two thermal dust components, suggesting that the majority of the gas/dust $\left(65 \pm 10 M_{\odot}\right)$ is at a relatively low temperature of $41 \pm 6 \mathrm{~K}$ corresponding to radiation peaked at a far-IR wavelength. There is an additional hot component $(\sim 400 \mathrm{~K})$ with little mass accounting for the secondary emission bump at mid-IR in the SED, suggesting that a small fraction of dust has been heated by newly born stars in the immediate surroundings.

The CARMA observations of the $\mathrm{HCO}^{+}(1-0)$ line confirmed the presence of blueshifted double-peak line profile toward the W3-SE core discovered by Wu et al. (2007) with the IRAM $30 \mathrm{~m}$. We made an attempt to fit the line profile with the infall model of Myers et al. (1996) by incorporating outflow components. The model fitting suggests that in addition to infalling gas, a significant contribution from outflows is necessary to produce the observed line profiles. The kinematics of the molecular gas in W3-SE observed with CARMA in the central 40" region are consistent with a bipolar outflow with a dynamic age of $\sim 3 \times 10^{4}$ yr. Finally, based on the observations with CARMA, SMA, and JCMT along with the previous published results, we conclude that W3-SE hosts a group of young stars and protostars which are likely located in the double dust cores observed with SMA.

We are grateful for the staff of the CARMA, SMA, and JCMT for their assistance in the observations. L.Z. thanks the organizers and tutors of the CARMA 2008 summer school, and J. Carpenter for his help in data reduction. We are grateful to $\mathrm{G}$. Ruch for providing the IRAC mosaic images and the point-source table, and the useful discussions with us on the infrared part of this work. L.Z. is supported by the SAO predoctoral program. L.Z. thanks the NRAO for providing him NSF travel fund to attend the millimeter/submillimeter conference in Taipei. Y.W. and L.Z. are grateful for the support of the grants 10873019 and 10733030 of NSFC.

\section{REFERENCES}

Arce, H. G., Shepherd, D., Gueth, F., Lee, C. F., Bachiller, R., Rosen, A., \& Beuther, H. 2007, in Protostars and Planets V, ed. B. Reipurth, D. Jewitt, \& K. Keil (Tucson, AZ: Univ. Arizona Press), 245

Attard, M., Houde, M., Novak, G., Li, H.-B., Vaillancourt, J., Dowell, C. D. Davidson, J., \& Shinnaga, H. 2009, ApJ, 702, 1584

Blitz, L., Fich, M., \& Stark, A. A. 1982, ApJS, 49, 183

Evans, N. J., II. 1999, ARA\&A, 37, 311

Fazio, G. G., et al. 2004, ApJS, 154, 10

Flaherty, K. M., Pipher, J. L., Megeath, S. T., Winston, E. M., Gutermuth, R. A., Muzerolle, J., Allen, L. E., \& Fazio, G. G. 2007, ApJ, 663, 1069

Hildebrand, R. H. 1983, QJRAS, 24, 267

Jijina, J., Myers, P. C., \& Adams, F. C. 1999, ApJS, 125, 161

Lada, C. J., Lombardi, M., \& Alves, J. F. 2009, ApJ, 703, 52

Makovoz, D., \& Khan, I. 2005, in ASP Conf. Ser. 347, Mosaicking with MOPEX, Astronomical Data Analysis Software and Systems XIV, ed. P. Shopbell, M. Britton, \& R. Ebert (San Francisco, CA: ASP), 81

Mardones, D., Myers, P. C., Tafalla, M., Wilner, D. J., Bachiller, R., \& Garay, G. 1997, ApJ, 489, 719

Myers, P. C., Mardones, D., Tafalla, M., Williams, J. P., \& Wilner, D. J. 1996, ApJ, 465, L133

Ossenkopf, \& Henning, T. 1994, A\&A, 291, 943

Pavlyuchenkov, Y., Wiebe, D., Schustov, B., Henning, T., Launhardt, R., \& Semenov, D. 2008, ApJ, 689, 335

Qin, S.-L., Zhao, J.-H., Moran, J. M., Marrone, D. P., Patel, N. A., Wang, J.-J., Liu, S.-Y., \& Kuan, Y.-J. 2008, ApJ, 677, 353

Rieke, G. H., et al. 2004, ApJS, 154, 25

Ruch, G. T., Jones, T. J., Woodward, C. E., Polomski, E. F., \& Gehrz, R. D. 2007, ApJ, 654, 338

Sault, R. J., Teuben, P. J., \& Wright, M. C. H. 1995, in ASP Conf. Ser. 77 Astronomical Data Analysis Software and Systems IV, ed. R. A. Shaw, H. E. Payne, \& J. J. E. Hayes (San Francisco, CA: ASP), 433

Scoville, N. Z., Sargent, A. I., Sanders, D. B., Claussen, M. J., Masson, C. R., Lo, K. Y., \& Phillips, T. G. 1986, ApJ, 303, 416

Shu, F. H. 1977, ApJ, 214, 488

Tieftrunk, A. R., Megeath, S. T., Wilson, T. L., \& Rayner, J. T. 1998, A\&A, 336,991

Wilson, T. L., Rohlfs, K., \& Hüttemeister, S. 2009, Tools of Radio Astronomy (5th ed.; Berlin: Springer)

Woods, R. C., Dixon, T. A., Saykally, R. J., \& Szanto, P. G. 1975, Phys. Rev. Lett., 35, 1269

Wu, Y., Henkel, C., Xue, R., Guan, X., \& Miller, M. 2007, ApJ, 669, L37

Zinchenko, I., Caselli, P., \& Pirogov, L. 2009, MNRAS, 395, 2234 